\title{
Effects of a brief, pedometer-based behavioral intervention for individuals with COPD during inpatient pulmonary rehabilitation on 6-week and 6-month objectively measured physical activity: study protocol for a randomized controlled trial
}

Wolfgang Geidl ${ }^{*}{ }^{*}$, Jana Semrau ${ }^{1}$, René Streber ${ }^{1}$, Nicola Lehbert ${ }^{2}$, Silke Wingart ${ }^{2}$, Alexander Tallner ${ }^{3}$, Michael Wittmann², Rupert Wagner ${ }^{2}$, Konrad Schultz ${ }^{2}$ and Klaus Pfeifer ${ }^{1}$

\begin{abstract}
Background: Pulmonary rehabilitation programs often fail to substantially enhance long-term physical activity in patients with chronic obstructive pulmonary disease (COPD). The reasons for successful physical activity changes in patients with COPD are not well understood. The need to better understand the determinants of physical activity in patients with COPD and effective rehabilitation strategies to improve physical activity is evident.

Methods/design: The STAR study (Stay Active after Rehabilitation) investigates, in a randomized controlled trial, the additional effect of a pedometer-based behavior-change intervention during inpatient pulmonary rehabilitation on objectively measured physical activity 6 weeks and 6 months post rehabilitation. The intervention uses the behavior-change techniques (1) instruction on how, where and when to perform the behavior, (2) prompt goal setting for physical activity, (3) prompt self-monitoring of behavior, and (4) feedback on behavior. The primary outcome of physical activity will be measured using a physical activity monitor (Actigraph wGT3X-BT) for a period of 7 days, firstly 2 weeks before rehabilitation begins (t0) as well as 6 weeks and 6 months after rehabilitation (t3, t4). Additionally, to predict physical activity progression after rehabilitation, a complex personal diagnostics battery, including questionnaires as well as functional assessments, is to be carried out at the start and end of rehabilitation (t1, t2). This battery is based on the foundational ideas of the Physical Activity-Related health Competence model.

Five hundred and two patients with COPD, aged 18 years or older and admitted for an approved pulmonary rehabilitation, will be enrolled in the STAR study.

(Continued on next page)
\end{abstract}

\footnotetext{
* Correspondence: wolfgang.geidl@fau.de

1 Institute of Sport Science and Sport, Friedrich-Alexander University

Erlangen-Nürnberg, Gebbertstr. 123b, 91058 Erlangen, Germany

Full list of author information is available at the end of the article
} 
(Continued from previous page)

Discussion: The STAR study is designed as a randomized controlled trial to gain a better understanding of the personal determinants of physical activity in patients with COPD and to evaluate a pedometer-based physical activity-change intervention in the context of inpatient pulmonary rehabilitation. The results enable the future identification of patients with COPD who will find it difficult to engage in long-term physical activity after rehabilitation. Based on this, intervention strategies to promote physical activity in the content of pulmonary rehabilitation can be optimized.

Trial registration: Clinicaltrials.gov, ID: NCT02966561. Registered retrospectively after the start of the recruitment in June 2016 on 22 November 2016. All protocol modifications will be registered in the trial registry.

Keywords: Behavior-change intervention, Chronic obstructive pulmonary disease (COPD), Implicit association test, Pedometer, Accelerometry, Physical activity-related health competence, Health-related quality of life (HRQOL)

\section{Background}

The initiation of a physically active lifestyle is a central goal of pulmonary rehabilitation (PR) [1]; though in patients with chronic obstructive pulmonary disease (COPD), PR programs often fail to substantially enhance long-term physical activity (PA) [2-4]. The reasons for successful PA behavior changes in patients with COPD are not well understood [5]. The role of psychological factors especially, and their interplay with physical functioning needs further investigation [6]. Consequently, following Spruit et al. [4], two aspects are needed for optimized patient treatment during PR: (1) a better understanding of the determinants of PA in patients with COPD and (2) effective strategies to improve important determinants and, accordingly, PA.

In covering the first point, the concept of Physical Activity-related health Competence (the PARC model) provides a new model to describe personal determinants of PA [7]. The PARC model focuses on personal competencies that favor the integration of PA in everyday life. The model's three components are movement competence, control competence, and self-regulation competence. These subcompetencies are directly linked to the requirements for initiating and maintaining PA with positive effects on health and wellbeing. In comparison to other health psychology models of health behavior, the competence-based perspective of the PARC model is considered to add value because it contains movementrelated, task-specific as well as sport-typical elements. Notably, the PARC model incorporates important individual physical and psychological characteristics. This is especially important for the explanation of PA behavior of older adults and clinical populations. The first validated, generic questionnaire for the subcompetencies of the PARC model is now available [7]. For clinical populations, this questionnaire could profit from an extension with indication-specific characteristics (e.g., obstructive lung function for patients with COPD), allowing for a complex and adequate assessment of individual determinants of PA in clinical populations.
With regard to the second point, the use of pedometers ranks as one of the most effective intervention elements for PA promotion [8]. Pedometers offer various possibilities to design behavior-change strategies [9]. In particular, pedometers are of use for persons to monitor and record their daily PA. Self-monitoring of behavior has been proven to be one of the most effective behaviorchange techniques (BCT) in clinical populations [10]. Furthermore, pedometers are suitable in support of other BCT [11], e.g., individual goal setting in the unit steps per day or giving feedback on behavior. In nonclinical populations, pedometer-based interventions achieve an average increased PA level of approximately 2000 steps per day (effect size $d=0.68$ ) in comparison to control groups [12]. Clinical populations provide preliminary evidence for the effectiveness of pedometers as part of rehabilitation for people with musculoskeletal disease [13], type-2 diabetes $[14]$ as well as COPD $[15,16]$. However, these results come from studies with small sample sizes in outpatient rehabilitation programs. Moreover, these studies often relate to short-term behavior changes during the rehabilitation period. The validity of most studies is further restricted by the usage of PA questionnaires instead of objective PA measurements [4]. Singh et al. [17] summarized that pedometer studies' sample sizes in PR were not, up to this point, suitable for the detection of meaningful differences in PA levels. In the context of inpatient PR the potential of pedometers to first initiate and afterward maintain a physically active lifestyle in the long term has not yet been investigated.

The purpose of this paper is to describe the design of a randomized controlled trial (RCT) to (1) evaluate the effect of a short, pedometer-based behavior-change intervention (BCI) for inpatient $\mathrm{PR}$ in COPD patients with regard to the initiation (6-week follow-up) and maintenance (6-month follow-up) of a physically active lifestyle after PR and (2) to predict the expected intervention treatment effect and PA progression after PR discharge utilizing an innovative diagnostic battery that is based on the PARC model [7]. 
The STAR study (Stay Active after Rehabilitation) aims to answer two central questions:

\#1: does the integration of a pedometer-based intervention during inpatient PR for people with COPD, in comparison to standard PR, lead to a sustained improvement of PR results with regards to PA 6 weeks and 6 months after the PR?

\#2: how does PA-related health competence of individuals with COPD develop during inpatient PR; and which individual PA-related health competence characteristics, in combination with which disease-specific prognostic assessments, enable the prediction of the expected intervention treatment effect as well as of the initiation and long-term maintenance of a physically active lifestyle after PR?

\section{Hypothesis}

\section{Primary hypothesis}

In individuals with COPD, inpatient PR (= standard care) plus a pedometer-based BCI will result in a significantly higher level of PA 6 weeks and 6 months after rehabilitation compared to standard care plus a short revision of patient education.

\section{Secondary hypothesis}

The secondary outcomes (PA-related health competence of patients with COPD in combination with other PA behavior-related psychological measurements and diseasespecific prognostic assessment) will enable the explanation of the treatment effect and the prediction of PA progression after PR discharge.

\section{Methods/design}

\section{Study design}

The study will be conducted using a randomized controlled intervention design with five measurement time points (t0-t4; see Fig. 1).

\section{Study population}

The study will take place within the German rehabilitation system which typically provides an inpatient rehabilitation in a disease-specific rehabilitation clinic for a duration of 3 weeks. Eligible are patients with COPD in all 2011 GOLD classifications A-D [18] and stages 1-4 who are enrolled for PR with the rehabilitation clinic Bad Reichenhall, the largest PR clinic in Germany. Recruitment starts in June 2016 and is likely to be finished in September 2017.

Table 1 provides an overview of all inclusion and exclusion criteria. The study nurse verifies inclusion criteria based on the written information available from the general practitioner who filed the application for PR. At the start of PR the admitting physician confirms the inclusion and exclusion criteria during the initial examination.

\section{Study flow and recruitment procedures}

This study consists of two phases (see Fig. 2). Phase I consists of the measurement point t0 2 weeks before the start of inpatient PR. Subjects in study phase I are asked to participate in phase II (t1-t4) upon arrival for PR.

Six weeks before the start of PR, eligible subjects will be contacted in writing by the PR clinic study nurse. The letter contains detailed information about the process of study phase I. Furthermore, patients are asked for their consent to be contacted via telephone. Persons who agree will be called by the study nurse, provided with further information about the study and asked to participate in study phase I. Participants return written informed consent via a prepaid envelope. This form provides the necessary consent for participation in the t0 measurement. The PA monitor will then be shipped out from the Erlangen study center. Enclosed along with the PA monitor, participants receive precise, illustrated instructions for wearing the PA monitor. Additional telephone support will be available from the Erlangen study center for all questions regarding study phase I, including any accelerometer issues.

Study phase II starts with the beginning of inpatient PR. During initial PR admission assessment subjects of study phase I will be informed about the course of the study phase II (t1-t4). A second signed informed consent is required for participation in phase II of the study. Subjects will then be randomized into two study arms, either the control group (CG) or the intervention group (IG). All subjects will first undergo an initial assessment of PA-related health competence (t1). Before the start of the study interventions (see the "Interventions" section below) in week 2 of the inpatient PR, CG and IG will each receive a short introductory session explaining the use of PA monitors. At the end of the inpatient PR, the assessment battery from $\mathrm{t} 1$ will be repeated ( $\mathrm{t} 2$ ).

\section{Follow-up phase}

The study center in Erlangen will contact subjects via letter 6 weeks (t3) and 6 months (t4) after discharge from the inpatient PR program. Provided that participant's current health status along with any other considerations (e.g., holidays) do not preclude wearing the PA monitor, the device and follow-up questionnaires will be sent to the subjects for t3-PA and t4-PA measurement. If the PA monitor and the questionnaire has not been returned within 2 weeks, postal reminders will be sent.

\section{Dropout criteria}

This study recruits patients with COPD assigned to PR. In some cases, after the physical examination in the 


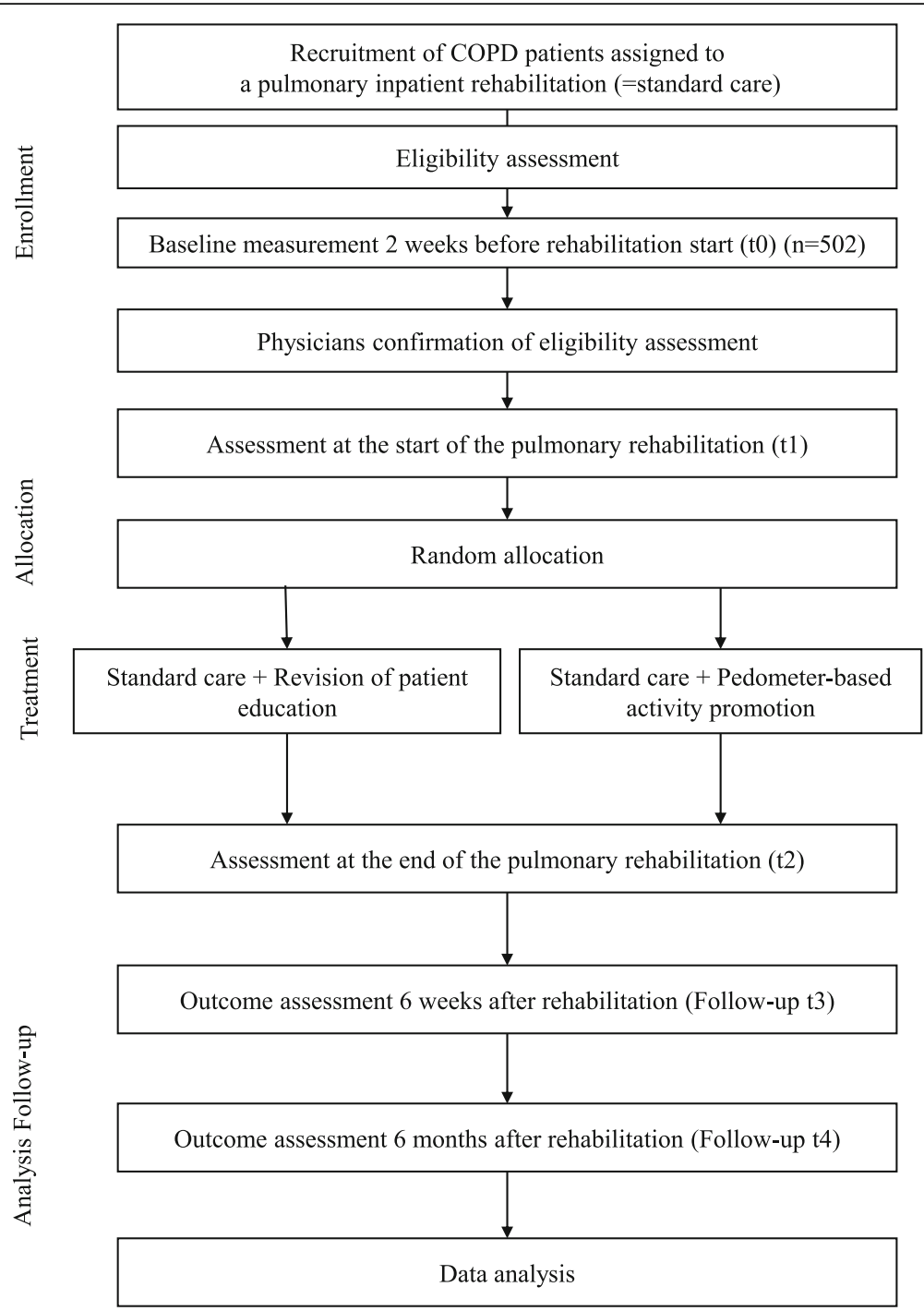

Fig. 1 Study flow chart of subjects

Table 1 Study inclusion and exclusion criteria

Inclusion criteria Main diagnosis for the pulmonary rehabilitation: (ICD-10) physician-confirmed diagnosis of chronic obstructive pulmonary disease (COPD) (International classification code: J44.- at all 2011 GOLD classifications A-D).

The diagnosis has to be confirmed in each case by lung function ( $\mathrm{FEV}_{1} \mathrm{NC}<0.7$ after bronchospasmolysis) and a respiratory specialist at admission to the pulmonary rehabilitation clinic

Age $\geq 18$ years

Exclusion criteria Severe comorbidities, which will affect the results of the outcome parameters much more than pulmonary rehabilitation; for example, cancer or severe cardiac or neurological comorbidities

Considerable reduction of sight and hearing

Severe psychiatric condition as a secondary diagnosis

Lack of ability to speak German

ICD-10 International Classification of Diseases, version 10 rehabilitation clinic, initial diagnoses are proven incorrect after already having completed the t0 measurement at home. These cases are not classified as dropouts but as being falsely included initially. Dropouts for study phase I are defined as patients who provided an informed consent for phase I but refused to complete the baseline evaluation (t0). Dropouts for study phase II are defined as patients who provided an informed consent for phase II but refused to complete one or more of the following measurements (t1-t4). Additionally, dropouts are classified as patients who withdraw from the study during phase I or phase II and prohibit the use of existing data. Subjects who did not complete treatment, or who did not complete assessment at the end of the inpatient PR but do not explicitly refuse or withdraw from the study, will be followed up. Figure 1 illustrates the flow chart of study subjects. 


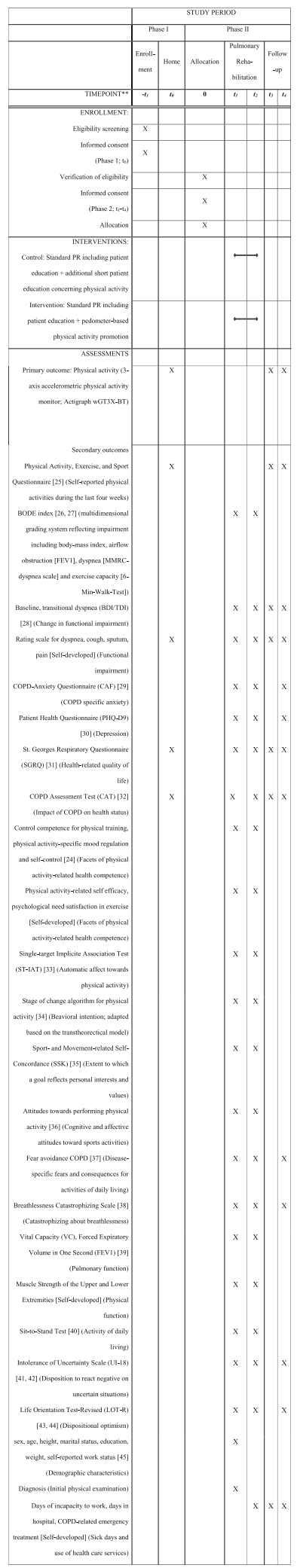

Fig. 2 Standard Protocol Items: Recommendation for Interventional Trials (SPIRIT) figure [37-57]

\section{Registration of nonresponders}

The registration of all eligible patients and the reporting of the number and sex of eligible patients who do not participate in the study will take place for both study phase I and study phase II.

\section{Randomization}

The randomization of subjects in study phase II is carried out by a study nurse after initial physical examination using an externally provided randomization list from the Friedrich-Alexander-Universität ErlangenNürnberg. Randomization is stratified based on the four prognostic dichotomous variables sex (male vs. female), progression of COPD (GOLD stages $1-2$ vs. stages $3-4$ ), age ( $<60$ years vs. $\geq 60$ years), and type of rehabilitation (PR in patients with stable COPD vs. PR after hospitalization due to subsequent COPD-related airway obstruction). The randomization list is created in blocks with six subjects per block. The study nurse is aware of the allocation in the different strata. The randomization scheme was generated by using the website Randomization.com.

\section{Sample size and power calculation}

A consecutive sample of 351 PR patients is needed. Pedometer-based interventions are among the most effective intervention components to promote PA in everyday life [8]. Meta-analysis has shown, in comparison to control groups, an average mean effect size of 0.68 on PA which represents an increase of 2000 steps per day [12]. The long-term effect on PA of standard inpatient PR in people with COPD is assumed to be small [16]. In comparison to standard PR we expect a small effect $(d=0.3)$ at t4. The power calculation (by means of software G*Power 3.0) with respect to the primary target (analysis of covariance to $t 4$, mean effect size of $d=0.3$, an alpha error level of $5 \%$, and statistical power of $80 \%$ ) results in a required sample of at least 351 subjects with complete data. In a preliminary pilot study, a generally high willingness to participate of $96 \%$ was recorded. A dropout of $30 \%$ from baseline measurement $\mathrm{t} 0$ to $\mathrm{t} 4 \mathrm{is}$ estimated based on the pilot study. With this dropout rate, the recruitment of a total of 502 subjects (251 subjects per group) is necessary. The study sample size is adequate for the determination of PA predictors after rehabilitation and with a weekly average of 12 subjects and a conservative recruitment rate of $66 \%$, the study aims should be reached within the recruiting period of 15 months.

\section{Interventions}

\section{Control arm}

The control group (CG) will receive standard PR. Standard $P R$ is an intensive and comprehensive inpatient PR in a specialized German rehabilitation clinic. This PR is described in further detail below. In addition, the CG 
receives a "behavior placebo" intervention during their PR. This intervention contains playful PA and revisions of information on PA (knowledge on exercise recommendations, knowledge on possibilities of self-regulation of endurance training exercise intensity) obtained earlier during patient education of the standard PR. Similar to the IG subjects, the CG receive a booklet that looks identical to the PA diary used in the IG. This booklet contains a repetition of PA-related information from the standard PR curriculum.

PR in the Bad Reichenhall Clinic. In Germany, PR is usually carried out as inpatient rehabilitation and generally lasts 3 weeks, an extension of 1 to 2 weeks is possible if necessary. PR is a comprehensive, multidisciplinary intervention based on an initial assessment followed by a combination of patient-tailored therapies $[1,19]$. In the Bad Reichenhall Clinic PR lasts an average of 25 days, is tailored to the patients' individual needs and includes the following obligatory main components: checking and, if required, adjusting the current COPD medication according to COPD guidelines; physical exercise (4-5 units/week of endurance exercise $(45 \mathrm{~min})$ and 3 units/week of strength exercise (45 min) per week, and 7 units/week whole-body vibration muscle training); structured COPD patient education ( $6 \mathrm{~h}$ of patient education COPD $+1 \mathrm{~h}$ inhaler device training), respiratory physiotherapy in groups (pursed lips breathing and other breathing and coughing techniques (2 units/week of $45 \mathrm{~min}$ )). Optional components include a comprehensive smoking-cessation program (at least 8 units), mucolytic physiotherapy, inspiratory muscle training (IMT), neuromuscular electrostimulation (NMES), saline inhalation therapy, psychological interventions and social counseling (individual and groups), nutritional counseling, patient education concerning long-term oxygen therapy, and occupational therapy including counseling on assistive devices. PR in Bad Reichenhall Clinic is implemented by an interdisciplinary rehabilitation team including experienced pneumologists who bear overall responsibility and coordinate the program along with further health professionals including physiotherapists, exercise therapists, occupational therapists, psychologists, social workers, nurses and nutritionists.

For all patients - IC and CG - receiving PR in the Bad Reichenhall Clinic, treatment includes several intervention components addressing the promotion of PA. Additional file 1 lists the behavior-change techniques $(\mathrm{BCT})$ included in standard care in the $\mathrm{Bad}$ Reichenhall Clinic according to the taxonomy of $\mathrm{BCT}$ from Michie et al. [11].

\section{Intervention arm}

Subjects in the intervention arm will receive the same standard inpatient PR program as the control arm.
Instead of the placebo intervention of the CG the IG will additionally receive a pedometer-based PA BCI.

The central components of the $\mathrm{BCI}$ include the following behavior-change techniques (BCT):

- Instruction on how, where and when to perform the behavior

- Prompt goal setting for PA

- Prompt self-monitoring of behavior

- Feedback on behavior

The BCI integrates the most frequently applied BCT for PA promotion in patients with COPD [20]. Additional file 2 explicates the sequence of the two $\mathrm{BCI}$ lessons and defines the used $\mathrm{BCT}$ with regard to the BCT taxonomy [11].

The BCI will consist of two 45-min sessions. The first lesson is placed in the end of the second week of PR and the second lesson will take place in the middle of the third week of PR. During lesson 1 of the BCI, subjects receive a pedometer and booklet containing a PA diary and PA-related information (recommendations for exercise and PA for patients with COPD). Subjects keep the pedometer and the booklet after their PR discharge. The intervention replaces standard exercise therapy and for this reason the total amount of therapy is identical. The $\mathrm{BCI}$ is delivered in open groups (varying composition of subjects possible) with six to 12 patients. An exercise therapist from the clinic will hold both lessons. As BCT included in the sessions relate to PA, the lessons take place in a gymnasium.

With regard to session length, placement $(2 \times 45 \mathrm{~min}$ in weeks 2 and 3 of the PR) and group composition, the CG lessons are identical to the IG.

\section{Outcome assessment}

\section{Primary outcome measures}

The primary outcome is objectively measured PA at 6 weeks (t3) and 6 months (t4) after PR. Baseline measurement of PA is conducted 2 weeks before the inpatient PR begins (T0). PA is measured on seven consecutive days with a three-axial accelerometer PA monitor (Actigraph wGT3X-BT). This activity monitor is the one of the most accurate devices for COPD patients and is explicitly recommended as a valid tool to measure PA in this clinical population [21-23].

\section{Secondary outcome measures}

Secondary outcome measures will assess central psychological and physical functional determinants of long-term health-promoting PA applicable to patients following PR. As defined by the PARC model [24] the following characteristics will be collected: physical motor functions and consequences of health problems 
in functioning (physical function and disease-related impairments in activities and participation), motivation and volition, sports-related goals and motives, stage of behavior change, as well as characteristics of health and wellbeing. Data will be entered and stored at the Erlangen Study Center. Outcomes of the study and time frames for their collection are shown in Fig. 2.

\section{Data analysis}

\section{Statistical analysis and evaluation}

Regarding the primary objective, a baseline-controlled comparison (covariance) of the post values between the initial assigned control and the intervention treatment group (intention-to-treat analysis) at the time points $\mathrm{t} 3$ and $t 4$ will be provided for statistical analysis.

For the secondary objective a cluster-based analysis method will be used for longitudinal records (e.g., linking of clusters after removal of a residue (LICUR); [25]). In this case the analysis creates separate clusters for each measurement using the comprehensive assessment battery for $\mathrm{t} 1$ and $\mathrm{t} 2$ and especially using the accelerometer PA data for $\mathrm{t} 0, \mathrm{t} 3$ and $\mathrm{t} 4$. The second step of the clusterbased analysis links separately created clusters in a longitudinal way.

A strategy for dealing with missing data will be developed in cooperation with a biostatistician. If adequate and realistic, missing data will be substituted by multiple imputation.

The processing of PA monitoring data as the primary outcome will be conducted as follows: the PA monitor will be worn for seven consecutive days with a minimum of four valid days inclusive of a weekend day [26-28]. A day is considered valid if subjects wear the device for ten or more waking hours [26-28], where non-wear time is set at $60 \mathrm{~min}$ of zero counts of which up to 2 min may be within the $0-100$ count range $[28,29]$. Current research points towards avoiding the use of direct monitor outputs such as energy expenditure estimations due to issues with reliability [21,30]. Consensus points towards the use of both sedentary and moderate to vigorous physical activity (MVPA) cutoff points where sedentary activity is seen as 0 to $>100$ counts per min [27, 29, 31, 32] and MVPA as $>1952$ counts per min [29]. While the use of the LFE filter to calculate steps is not recommended, it may be applicable during data analysis of cutoff points for older adults [33, 34]. Future work on defining cutoff points in clinical populations will be considered.

\section{Blinding}

Blinding of the therapists was not possible as they work in the same clinical team and are involved in the development of this study. Patients will be masked with regard to study group. They will be informed by staff and in the "informed consent" that the effectiveness of two exercise therapy programs will be compared and that both meet current scientific standards and are appropriate to improve health status. During the study period, patients are not informed as to whether they participate in the control or the intervention group. A research assistant not involved in the study process will perform the statistical data analysis.

\section{Discussion}

A better understanding of the determinants of PA in patients with COPD and effective PR strategies to improve PA is evident. The STAR study fills two gaps in the academic literature (1) it investigates the ability of PR to promote PA to patients with COPD in the long term and (2) it investigates COPD individuals' personal determinants of (un-)successful PA changes.

The RCT design allows the evaluation of a short, pedometer-based $\mathrm{BCI}$ for inpatient PR in COPD patients with regard to the initiation (6-week follow-up) and maintenance (6-month follow-up) of a physically active lifestyle after PR. The design is suitable to overcome restrictions in previous empirical findings due to several reasons: it contains an adequately powered sample size, objectively measures PA with a validated measurement tool, and collects baseline PA data before PR in study phase I as well as at the 6-month follow-up after PR (study phase II). The reason for the two-phased study design is not to overburden eligible participants during initial recruitment and thereby ensure higher participation rates. Adding an additional 12-month follow-up would detect enduring effects on PA thereby further strengthening the study design. Nevertheless, the academic literature indicates that PA status of COPD patients a few months and 1 year after PR is unlikely to differ $[35,36]$. If the inexpensive, pedometerbased intervention proves its effectiveness it may be easily applied to similar inpatient rehabilitation programs. These findings may then also apply to other clinical populations.

Furthermore, the study generates a better understanding as to why patients with COPD manage or fail to initiate and maintain PA after PR. Based on the concepts from the model of PA-related health competence (PARC model) $[7,24]$ an adequate setup of individual physical and psychological determinants of PA will be assessed. The diagnostic battery will enrich our understanding of why the pedometer-based intervention works. In particular, the PA-related regulation competence is expected to contribute to this explanation of the intervention treatment effect. Furthermore, the results of a clusterbased analysis enable, regardless of the randomly controlled pedometer intervention's effectiveness, the future identification of patients with COPD who will find it difficult to engage in long-term, health-promoting PA after PR. Acquiring a valid model for the prediction of PA 
behavior is the basis for optimizing intervention strategies to promote PA in the context of PR.

\section{Trial status}

The STAR study started in June 2016 and completion of recruitment is estimated for September.

\section{Additional files}

Additional file 1: Promoting physical activity: behavior change techniques used during Pulmonary Rehabilitation standard care in the Clinic Bad Reichenhall. (DOCX 15 kb)

Additional file 2: Content of the two lessons of the pedometer-based physical activity (PA) behavior-change interventions ( $\mathrm{BCl}$ ) classified with the taxonomy of behavior change techniques from Michie et al. [11]. (DOCX $17 \mathrm{~kb}$ )

Additional file 3: SPIRIT 2013 Checklist: recommended items to address in a clinical trial protocol and related documents. (DOC $102 \mathrm{~kb}$ )

\section{Abbreviations}

$\mathrm{BCl}$ : Behavior-change intervention; $\mathrm{BCT}$ : Behavior-change technique; CG: Control group; COPD: Chronic obstructive pulmonary disease; IG: Intervention group; PA: Physical activity; PARC model: Physical Activity-related health Competence model; PR: Pulmonary rehabilitation

\section{Acknowledgements}

The authors thank the German Pension Insurance Association, Section Bavaria South (Deutsche Rentenversicherung Bayern Süd), which sponsors and supports this study. We thank Amone Leithäusser who is the head of the section physical therapy, and the entire team of physical therapists, the study nurses Stephanie Häusl, Andrea Klotz, Maike Messerschmidt, Berta Obermaier and the recruiting physicians of the Bad Reichenhall Clinic for their outstanding engagement. We further thank Samuel Cassar for the language revision of this manuscript. We acknowledge support from the Deutsche Forschungsgemeinschaft and Friedrich-Alexander-Universität Erlangen-Nürnberg (FAU) within the funding program Open Access Publishing.

\section{Funding}

This study is founded by the German Pension Insurance Association, Section Bavaria South (Deutsche Rentenversicherung Bayern Süd; Abteilung Rehabilitation und Sozialmedizin, Am Alten Viehmarkt 2, 84028 Landshut, Germany) (Reference number: 5.011-6.031.115) (www.deutscherentenversicherung-bayernsued.de) which was not involved in the design of the study and collection, management, analysis, interpretation of data, in writing the manuscript, or the decision to submit the report for publication.

\section{Availability of data and materials}

WG, KS, and KP will have access to the final trial dataset.

\section{Declarations}

The SPIRIT (Standard Protocol Items: Recommendations for Interventional Trials) Statement is included as Additional file 3.

\section{Authors' contributions}

WG, KS, JS, RS, AT, NL, SW, MW, RW, and KP conceived and designed the study. NL and SW will be responsible for the delivering of the intervention. MW, RW, and KP will be responsible for the recruiting. WG, KS, and KP are the principal investigators and will analyze the data. WG wrote the paper. WG will respond to public and scientific queries. All authors edited, revised and approved the final manuscript.

\section{Ethics approval and consent to participate}

The study protocol was approved by the independent Research Ethics Committee of the Medical Faculty of Friedrich-Alexander-Universität Erlangen-Nürnberg, Germany in 2015 (Ref. No. 321_15B).
The Department of Data Privacy Collection of the German Pension Insurance Association, Section Bavaria South approved the STAR study data security model to ensure confidentiality before, during, and after the trial. Signed informed consent of the participants is a prerequisite for taking part in the STAR study.

\section{Consent for publication}

Not applicable

\section{Competing interests}

The authors declare that they have no competing interests.

\section{Publisher's Note}

Springer Nature remains neutral with regard to jurisdictional claims in published maps and institutional affiliations.

\section{Author details}

${ }^{1}$ Institute of Sport Science and Sport, Friedrich-Alexander University Erlangen-Nürnberg, Gebbertstr. 123b, 91058 Erlangen, Germany. ${ }^{2}$ Klinik Bad Reichenhall, Centre for Rehabilitation, Pulmonology and Orthopaedics, Salzburger Straße 8-11, 83435 Bad Reichenhall, Germany. ${ }^{3}$ MedDay Pharmaceuticals GmbH, Münsterstraße 5, 59065 Hamm, Germany.

Received: 7 December 2016 Accepted: 27 July 2017

Published online: 29 August 2017

References

1. Spruit MA, Singh SJ, Garvey C, ZuWallack R, Nici L, Rochester C, et al. An official American Thoracic Society/European Respiratory Society statement: key concepts and advances in pulmonary rehabilitation. Am J Respir Crit Care Med. 2013;188:e13-64. doi:10.1164/rccm.201309-1634ST

2. Busby AK, Reese RL, Simon SR. Pulmonary rehabilitation maintenance interventions: a systematic review. Am J Health Behav. 2014;38:321-30. doi: 10.5993/AJHB.38.3.1

3. Ng LWC, Mackney J, Jenkins S, Hill K. Does exercise training change physical activity in people with COPD? A systematic review and meta-analysis. Chron Respir Dis. 2012:9:17-26. doi:10.1177/1479972311430335.

4. Spruit MA, Pitta F, McAuley E, Zuwallack RL, Nici L. Pulmonary rehabilitation and physical activity in patients with COPD. Am J Respir Crit Care Med. 2015;192:924-33. doi:10.1164/rccm.201505-0929Cl.

5. Gimeno-Santos E, Frei A, Steurer-Stey C, de Batlle J, Rabinovich RA, Raste $Y$, et al. Determinants and outcomes of physical activity in patients with COPD: a systematic review. Thorax. 2014;69:731-9. doi:10. 1136/thoraxinl-2013-204763.

6. Altenburg WA, ten Hacken NHT, Bossenbroek L, Kerstjens HA, de Greef MHG, Wempe JB. Short- and long-term effects of a physical activity counselling programme in COPD: a randomized controlled trial. Respir Med. 2015:109:112-21. doi:10.1016/.rmed.2014.10.020.

7. Sudeck G, Pfeifer K. Physical activity-related health competence as an integrative objective in exercise therapy and health sports-conception and validation of a short questionnaire. Sportwiss. 2016;46:74-87. doi:10.1007/ s12662-016-0405-4.

8. Heath GW, Parra DC, Sarmiento OL, Andersen LB, Owen N, Goenka S, et al. Evidence-based intervention in physical activity: lessons from around the world. Lancet. 2012;380:272-81. doi:10.1016/S0140-6736(12)60816-2 .

9. Koring M, Parschau L, Lange D, Fleig L, Knoll N, Schwarzer R. Preparing for physical activity: pedometer acquisition as a self-regulatory strategy. Appl Psychol Health Well Being. 2013;5:136-47.

10. Conn VS, Hafdahl AR, Brown SA, Brown LM. Meta-analysis of patient education interventions to increase physical activity among chronically ill adults. Patient Educ Couns. 2008;70:157-72. doi:10.1016/j.pec.2007.10.004

11. Michie S, Richardson M, Johnston M, Abraham C, Francis J, Hardeman W, et al. The behavior change technique taxonomy (v1) of 93 hierarchically clustered techniques: building an international consensus for the reporting of behavior change interventions. Ann Behav Med. 2013;46:81-95. doi:10. 1007/s12160-013-9486-6.

12. Kang M, Marshall SJ, Barreira TV, Lee J. Effect of pedometer-based physical activity interventions: a meta-analysis. Res Q Exerc Sport. 2009:80:648-55.

13. Mansi S, Milosavljevic S, Baxter G, Tumilty S, Hendrick P. A systematic review of studies using pedometers as an intervention for musculoskeletal diseases. BMC Musculoskelet Disord. 2014;15:231. doi:10.1186/1471-2474-15-231. 
14. Funk M, Laurette-Taylor E. Pedometer-based walking interventions for free-living adults with type 2 diabetes: a systematic review. Curr Diabetes Rev. 2013;9:462-71.

15. Mendoza L, Horta P, Espinoza J, Aguilera M, Balmaceda N, Castro A, et al. Pedometers to enhance physical activity in COPD: a randomised controlled trial. Eur Respir J. 2014. doi:10.1183/09031936.00084514

16. Bertici N, Fira-Mlǎdinescu O, Oancea C, Tudorache $V$. The usefulness of pedometry in patients with chronic obstructive pulmonary disease. Multidiscip Respir Med. 2013;8(1):7.

17. Singh S, Morgan M. One step beyond, does rehabilitation influence physical activity? Chron Respir Dis. 2012;9:3-4. doi:10.1177/1479972311432354 .

18. Vestbo J, Hurd SS, Agustí AG, Jones PW, Vogelmeier C, Anzueto A, et al. Global strategy for the diagnosis, management, and prevention of chronic obstructive pulmonary disease GOLD executive summary. Am J Respir Crit Care Med. 2013:187:347-65. doi:10.1164/rccm.201204-0596PP.

19. Fischer J, Schnabel M, Sitter H. Rehabilitation of patients with chronic obstructive pulmonary disease (COPD) S2 guideline of the German Society for Pneumology and Respiratory Medicine and the German Society for Rehabilitation Science (RGRW). Pneumologie. 2007;61:233-48. doi:10.1055/ s-2007-959196.

20. Wilson JJ, O'Neill B, Collins EG, Bradley J. Interventions to increase physical activity in patients with COPD: a comprehensive review: COPD: Journal of Chronic Obstructive Pulmonary Disease. COPD. 2015;12:332-43. doi:10.3109/ 15412555.2014.948992.

21. Rabinovich RA, Louvaris Z, Raste $Y$, Langer D, van Remoortel H, Giavedoni S, et al. Validity of physical activity monitors during daily life in patients with COPD. Eur Respir J. 2013;42:1205-15. doi:10.1183/09031936.00134312.

22. van Remoortel H, Raste Y, Louvaris Z, Giavedoni S, Burtin C, Langer D, et al. Validity of six activity monitors in chronic obstructive pulmonary disease: a comparison with indirect calorimetry. PLoS One 2012;7. doi:10.1371/journal. pone.0039198

23. Strath SJ, Pfeiffer KA, Whitt-Glover MC. Accelerometer use with children, older adults, and adults with functional limitations. Med Sci Sports Exerc. 2012;44:S77-85. doi:10.1249/MSS.0b013e3182399eb1.

24. Pfeifer K, Sudeck G, Geidl W, Tallner A. Bewegungsförderung und Sport in der Neurologie - Kompetenzorientierung und Nachhaltigkeit. Neurol Rehabil. 2013;19;1;7-19.

25. Bergman LR, Wångby $M$. The person-oriented approach. A short theoretical and practical guide. Est J Educat. 2014;2. doi:10.12697/eha.2014.2.1.02b.

26. Matthews $C E$, Hagströmer M, Pober DM, Bowles HR. Best practices for using physical activity monitors in population-based research. Med Sci Sports Exerc. 2012:44:S68-76. doi:10.1249/MSS.0b013e3182399e5b.

27. Pedišić Z, Bauman A. Accelerometer-based measures in physical activity surveillance: Current practices and issues. Br J Sports Med. 2015;49:219-23. doi:10.1136/bjsports-2013-093407.

28. Thiel C, Gabrys L, Vogt L. Measuring physical activity with wearable accelerometers. Dtsch Z Sportmed. 2016:67:44-8. doi:10.5960/dzsm.2016.220

29. Gorman E, Hanson HM, Yang PH, Khan KM, Liu-Ambrose T, Ashe MC. Accelerometry analysis of physical activity and sedentary behavior in older adults: a systematic review and data analysis. Eur Rev Aging Phys Act. 2014; 11:35-49. doi:10.1007/s11556-013-0132-x.

30. van Remoortel H, Camillo CA, Langer D, Hornikx M, Demeyer H, Burtin C, et al. Moderate intense physical activity depends on selected metabolic equivalent of task (MET) cut-off and type of data analysis. PLoS One. 2013;8: e84365. doi:10.1371/journal.pone.0084365.

31. Evenson KR, Wen F, Metzger JS, Herring AH. Physical activity and sedentary behavior patterns using accelerometry from a national sample of United States adults. Int J Behav Nutr Phys Act. 2015;12:20. doi:10.1186/s12966-015-0183-7.

32. Wallén MB, Nero H, Franzén E, Hagströmer M. Comparison of two accelerometer filter settings in individuals with Parkinson's disease. Physiol Meas. 2014;35:2287-96. doi:10.1088/0967-3334/35/11/2287.

33. Korpan SM, Schafer JL, Wilson K, Webber SC. Effect of actigraph GT3X+ position and algorithm choice on step count accuracy in older adults. J Aging Phys Act. 2015;23:377-82. doi:10.1123/japa.2014-0033.

34. Lee I, Shiroma EJ. Using accelerometers to measure physical activity in large-scale epidemiological studies: issues and challenges. Br J Sports Med. 2014;48:197-201. doi:10.1136/bjsports-2013-093154.

35. Soicher J, Mayo E, Gauvin L, Hanley J, Bernard S, Maltais F, et al. Trajectories of endurance activity following pulmonary rehabilitation in COPD patients. Eur Respir J. 2012;39:272-8. doi:10.1183/09031936.00026011.
36. Saunders T, Dechman G, Hernandez P, Spence J, Rhodes R, McGannon K, et al. Distinct trajectories of physical activity among patients with COPD during and after pulmonary rehabilitation. COPD. 2015;12:539-45. doi:10.3109/15412555. 2014.995286.

37. Fuchs R, Klaperski S, Gerber M, Seelig H. Messung der Bewegungs- und Sportaktivität mit dem BSA-Fragebogen. Z Gesundheitspsychol. 2015;23:60-76. doi:10.1026/0943-8149/a000137.

38. Celli BR, Cote CG, Marin JM, Casanova C, Montes De Oca M, Mendez RA, et al. The body-mass index, airflow obstruction, dyspnea, and exercise capacity index in chronic obstructive pulmonary disease. N Engl J Med. 2004;350:1005-12.

39. Puhan MA, Garcia-Aymerich J, Frey M, ter Riet G, Antó JM, Agustí AG, et al. Expansion of the prognostic assessment of patients with chronic obstructive pulmonary disease: the updated BODE Index and the ADO Index. Lancet. 2009;374:704-11.

40. Mahler DA, Weinberg DH, Wells CK, Feinstein AR. The measurement of dyspnea. Contents, interobserver agreement, and physiologic correlates of two new clinical indexes. Chest. 1984:85:751-8.

41. Kühl K, Kuhn C, Kenn K, Rief W. Der COPD-Angst-Fragebogen (CAF): Ein neues Instrument zur Erfassung krankheitsspezifischer Ängste bei COPD-Patienten. Psychother Psychosom Med Psychol. 2011;61:e1-9. doi:10. 1055/s-0030-1248281.

42. Löwe B, Spitzer R, Zipfel S, Herzog W. Gesundheitsfragebogen für Patienten (PHQ D). Komplettversion und Kurzform. Testmappe mit Manual, Fragebögen, Schablonen. 2. Auflage. Karlsruhe: Pfizer; 2002.

43. Jones PW, Quirk FH, Baveystock CM, Littlejohns P. A self-complete measure of health status for chronic airflow limitation. The St. George's Respiratory Questionnaire. Am Rev Respir Dis. 1992;145:1321-7.

44. Ardelean DL, lulia L, Popescu R, Didilescu C, Dinescu S, Olteanu M, Nitu M. Evaluation of COPD patients using CAT-COPD assessment test. Pneumologia. 2012;61:221-9.

45. Bluemke M, Friese M. Reliability and validity of the Single-Target IAT (ST-IAT): assessing automatic affect towards multiple attitude objects. Eur J Soc Psychol. 2008;38:977-97. doi:10.1002/ejsp.487.

46. Huber $G$, Sudeck $G$. Entwicklung einer person-orientierten Bewegungstherapie in der medizinischen Rehabilitation. Unveröffentlichter Abschlussbericht des von der Deutschen Rentenversicherung Bund geförderten Projektes Nr. 0422-40-64-50-16; available online: http:// forschung.deutsche-rentenversicherung.de/ForschPortalWeb/ressource?key= Final2014_Abschlussbericht_PersonOrientierteBewegungstherapie.pdf

47. Seelig $H$, Fuchs $R$. Messung der sport- und bewegungsbezogenen Selbstkonkordanz. Z Sportpsychol. 2006;13:121-39. doi:10.1026/1612-5010. 13.4.121.

48. Brand R. Die affektive Einstellungskomponente und ihr Beitrag zur Erklärung von Sportpartizipation. Z Sportpsychol. 2006;13:147-55. doi:10.1026/16125010.13.4.147.

49. Stenzel N, Rief W, Kenn K. Fear Avoidance-Eine bedeutsame Aktivitätsbremse bei chronisch obstruktiver Lungenerkrankung (COPD)? Vortrag präsentiert auf dem 54. Kongress der Deutschen Gesellschaft für Pneumologie und Beatmungsmedizin (DGP), Hannover. 2013.

50. Solomon BK, Wilson KG, Henderson PR, Poulin PA, Kowal J, McKim DA. A breathlessness catastrophizing scale for chronic obstructive pulmonary disease. J Psychosom Res. 2015;79:62-8. doi:10.1016/j.jpsychores.2014.11.020.

51. Ruppel GL, Enright PL. Pulmonary function testing. Respir Care. 2012;57:16575. doi:10.4187/respcare.01640.

52. Jones SE, Kon SSC, Canavan JL, Patel MS, Clark AL, Nolan CM, et al. The fiverepetition sit-to-stand test as a functional outcome measure in COPD. Thorax. 2013;68:1015-20. doi:10.1136/thoraxjnl-2013-203576.

53. Gerlach AL, Andor T, Patzelt J. Die Bedeutung von Unsicherheitsintoleranz für die Generalisierte Angststörung Modellüberlegungen und Entwicklung einer deutschen Version der Unsicherheitsintoleranz-Skala. Z Klin Psychol Psychother. 2008;37:190-9. doi:10.1026/1616-3443.37.3.190

54. Carleton RN, Norton MPJ, Asmundson GJ. Fearing the unknown. A short version of the intolerance of uncertainty scale. J Anxiety Disord. 2007;21: 105-17. doi:10.1016/j.janxdis.2006.03.014.

55. Glaesmer H, Hoyer J, Klotsche J, Herzberg PY. Die deutsche Version des Life-Orientation-Tests (LOT-R) zum dispositionellen Optimismus und Pessimismus. Z Gesundheitspsychol. 2008;16:26-31. doi:10.1026/09438149.16.1.26.

56. Scheier MF, Carver CS, Bridges MW. Distinguishing optimism from neuroticism (and trait anxiety, self-mastery, and self-esteem): a reevaluation of the life orientation test. J Pers Soc Psychol. 1994;67:1063-78. 
57. Deck R, Röckelein. Zur Erhebung soziodemographischer und sozialmedizinischer Indikatoren in den rehabilitationswissenschaftlichen Forschungsverbünden. Förderschwerpunkt "Rehabilitationswissenschaften". Empfehlungen der Arbeitsgruppen"Generische Methoden", "Routinedaten" und "Reha-Ökonomie". Verband Deutscher Rentenversicherungsträger (Hrsg.), Förderschwerpunkt "Rehabilitationswissenschaften". Empfehlungen der Arbeitsgruppen "Generische Methoden", "Routinedaten" und "Reha-Ökonomie". DRV-Schriften. 1999;16:84-102.

Submit your next manuscript to BioMed Central and we will help you at every step:

- We accept pre-submission inquiries

- Our selector tool helps you to find the most relevant journal

- We provide round the clock customer support

- Convenient online submission

- Thorough peer review

- Inclusion in PubMed and all major indexing services

- Maximum visibility for your research

Submit your manuscript at www.biomedcentral.com/submit
Biomed Central 